\title{
ON DUALS OF SMOOTH PLANE CURVES
}

\author{
MASAAKI HOMMA \\ (Communicated by Louis J. Ratliff, Jr.)
}

\begin{abstract}
We prove that for given two distinct smooth curves in the projective plane over an algebraically closed field, their duals in the dual plane coincide if and only if they are conics in characteristic two with the same center.
\end{abstract}

\section{INTRODUCTION}

In the theory of plane projective geometry in positive characteristic, we are cognizant of many nonclassical phenomena of tangent lines of plane curves. One of the most surprising phenomena is the following fact proved by Wallace [10]. (His discussion was refined by Kleiman and Thorup [7].) Let $\mathbb{P}^{2}$ be a projective plane over an algebraically closed field of characteristic $p>0$ and $\check{\mathbb{P}}^{2}$ the dual plane of $\mathbb{P}^{2}$. For any given curve in $\check{\mathbb{P}}^{2}$, any given positive power $q$ of $p$, and any given positive integer $s$, there exists a curve $C \subset \mathbb{P}^{2}$ such that the dual $C^{*}$ of $C$ coincides with the assigned curve and the Gauss map $\gamma: C \rightarrow C^{*}$ has separable degree $s$ and inseparable degree $q$.

In the above situation, if one desires to choose $C$ as smooth, $s$ must be 1 by Kaji's theorem [6]. Moreover, if the assigned curve is a line, then $q=p=2$ by Lluis's theorem [9].

In his expository work on recent progress on plane curves in characteristic $p$ [8], Kleinman asks, "Is every curve of degree at least two the dual of a smooth curve whose Gauss map has any given inseparable degree $q$ ?"

When the assigned curve in question is smooth, we can see that the answer is negative by observing the list of smooth curves having smooth duals $[4,(6.6)$, (6.7)]. So a small modification should be necessary for the question, that is, we should assume the assigned curve to be singular.

Our results are motivated by the question and show the answer to be negative, even if the question is made into such a modification.

Theorem 1. Let $C_{1}$ and $C_{2}$ be distinct smooth curves in $\mathbb{P}^{2}$. Then $C_{1}^{*}=C_{2}^{*}$ in $\breve{\mathbb{P}}^{2}$ if and only if $C_{1}$ and $C_{2}$ are conics in characteristic two with the same center.

Received by the editors November 25, 1991.

1991 Mathematics Subject Classification. Primary 14N05; Secondary 14H35.

Key words and phrases. Nonreflexive curve, dual curve, Gauss map. 
Theorem 2. Let $d^{*}$ be a prime number and $C$ a smooth plane curve. Then $\operatorname{deg} C^{*}=d^{*}$ if and only if either

(1) $d^{*}=2, p \neq 2$, and $C$ is a conic;

(2) $d^{*}=3, p=2$, and $C$ is a cubic; or

(3) $d^{*}$ is of form $2^{e}+1(e>0), p=2$, and $C$ is projectively equivalent to the curve $X^{2^{e}+1}+Y^{2^{e}+1}+Z^{2^{e}+1}=0$.

Kaji's theorem and the standard Plücker formula (for smooth $C$ ) are essential tools for our studies.

Kaji's theorem. Let $C$ be a smooth plane curve. Then the Gauss map $\gamma: C \rightarrow C^{*}$ is purely inseparable.

Proof. See [6, (4.5)].

Plücker formula. Let $C$ be a smooth plane curve of degree $d$ with $\operatorname{deg} C^{*}=d^{*}$. Then we have $q d^{*}=d(d-1)$, where $q$ is the (inseparable) degree of the Gauss map $\gamma: C \rightarrow C^{*}$.

Proof. By definition, we have $\gamma^{*} \mathscr{O}_{C^{*}}(1) \cong \mathscr{O}_{C}(d-1)$. Calculate the degree of the invertible sheaf in two ways and use Kaji's theorem. Then we have the formula.

\section{SMOoth Plane CUbics in CHARACTERISTIC TWO}

First we study smooth plane cubics in characteristic two. The result of this section is used in the proof of Theorem 1, which will be given in $\S 2$.

Proposition 3. Let $E$ be a smooth cubic in $\mathbb{P}^{2}$ over an algebraically closed field of characteristic two. Then $E^{*}$ is a smooth cubic and, hence, $E^{* *}$ is too. Moreover, the composition of Gauss maps $\gamma^{*} \circ \gamma: E \rightarrow E^{* *} \subset \check{\mathbb{P}}^{2}=\mathbb{P}^{2}$ sends the flexes of $E$ onto those of $E^{* *}$, where $\gamma^{*}$ is the Gauss map of $E^{*}$.

Proof. Thanks to a theorem of Deuring [2], we can start from a simple defining equation of $E$, that is, $E$ is projectively equivalent to a curve defined by the equation

$$
F= \begin{cases}X^{3}+Y^{2} Z+Y Z^{2}=0 & \text { if } j=j(E)=0 \\ j X^{3}+X Y^{2}+X Y Z+Z^{3} / j=0 & \text { if } j=j(E) \neq 0\end{cases}
$$

where $j$ is the $j$-invarant of $E$.

The first part of our assertion was proved in [4, (6.7)]. (Note that there is a small mistake in that proof; $V=\partial F / \partial Y$ and $W=\partial F / \partial Z$ should read as $V=\partial F / \partial Z$ and $W=\partial F / \partial Y$, respectively.) Putting $U=\partial F / \partial X$, $V=\partial F / \partial Z$, and $W=\partial F / \partial Y$, a defining equation of the dual $E^{*}$ of $E$ is

$$
\begin{cases}U^{3}+V^{2} W+V W^{2}=0 & \text { if } j(E)=0, \\ U V^{2}+U V W+U^{2} W / j+W^{3}=0 & \text { if } j(E) \neq 0 .\end{cases}
$$

If $j=0$, then $\gamma(x, y, z)=\left(x^{2}, y^{2}, z^{2}\right)$ for any $(x, y, z) \in E$. Hence $\gamma: E \rightarrow E^{*}$ can be extended to a purely inseparable morphism $\mathbb{P}^{2} \rightarrow \check{\mathbb{P}}^{2}$. Observing the equation of $E^{*}$ and using the above fact, $\gamma^{*}: E^{*} \rightarrow E^{* *}$ is also extendable to a purely inseparable morphism $\check{\mathbb{P}}^{2} \rightarrow \check{\mathbb{P}}^{2}=\mathbb{P}^{2}$; therefore, $\gamma^{*} \circ \gamma: E \rightarrow E^{* *}$ can be extended to a Frobenius morphism $\mathbb{P}^{2} \rightarrow \mathbb{P}^{2}$ and hence sends the flexes of $E$ onto those of $E^{* *}$. 
Now we consider the case $j(E) \neq 0$. Change coordinates of $\check{\mathbb{P}}^{2}$ as

$$
\left(\begin{array}{c}
U \\
V \\
W
\end{array}\right)=\left(\begin{array}{ccc}
j^{2} & 0 & 0 \\
j & 1 & 0 \\
0 & 0 & 1
\end{array}\right)\left(\begin{array}{c}
U_{1} \\
V_{1} \\
W_{1}
\end{array}\right) .
$$

Then $E^{*}$ is defined by the equation

$$
G=j^{2} U_{1}^{3}+U_{1} V_{1}^{2}+U_{1} V_{1} W_{1}+W_{1}^{3} / j^{2}=0 .
$$

Putting $X^{\prime}=\partial G / \partial U_{1}, \quad Y^{\prime}=\partial G / \partial W_{1}$, and $Z^{\prime}=\partial G / \partial V_{1}$ and changing coordinates of $\check{\mathbb{P}}^{2}=\mathbb{P}^{2}$ to

$$
\left(\begin{array}{l}
X^{\prime} \\
Y^{\prime} \\
Z^{\prime}
\end{array}\right)=\left(\begin{array}{lll}
j^{4} & 0 & 0 \\
j^{2} & 1 & 0 \\
0 & 0 & 1
\end{array}\right)\left(\begin{array}{l}
\bar{X} \\
\bar{Y} \\
\bar{Z}
\end{array}\right),
$$

an equation of $E^{* *}$ is

$$
H=j^{4} \bar{X}^{3}+\overline{X Y}^{2}+\overline{X Y Z}+\bar{Z}^{3} / j^{4}=0 .
$$

Now we compute $\gamma^{*} \circ \gamma(x, y, z)$ for $(x, y, z) \in E$. We denote coordinates of points which are the image of $(x, y, z)$ by small letters corresponding to capital letters of the frame of reference.

Hence we have

$$
\left\{\begin{array}{l}
\bar{x}=\frac{1}{j^{4}} x^{\prime}=\frac{1}{j^{2}} u_{1}^{2}+\frac{1}{j^{4}} v_{1}^{2}+\frac{1}{j^{4}} v_{1} w_{1}, \\
\bar{y}=\frac{1}{j^{2}} x^{\prime}+y^{\prime}=u_{1}^{2}+\frac{1}{j^{2}} v_{1}^{2}+\frac{1}{j^{2}} v_{1} w_{1}+u_{1} v_{1}+\frac{1}{j^{2}} w_{1}^{2}, \\
\bar{z}=z^{\prime}=u_{1} w_{1}
\end{array}\right.
$$

and

$$
\left\{\begin{array}{l}
u_{1}=\frac{1}{j^{2}} u=\frac{1}{j} x^{2}+\frac{1}{j^{2}} y^{2}+\frac{1}{j^{2}} y z, \\
v_{1}=\frac{1}{j} u+v=x^{2}+\frac{1}{j} y^{2}+\frac{1}{j} y z+x y+\frac{1}{j} z^{2}, \\
w_{1}=w=x z .
\end{array}\right.
$$

Using the equation $j x^{3}+x y^{2}+x y z+z^{3} / j=0$, we have $\bar{x}=x^{4} / j^{3}, \bar{y}=y^{4} / j^{3}$, and $\bar{z}=z^{4} / j^{3}$. This means that $\gamma^{*} \circ \gamma: E \rightarrow E^{* *}$ can be extended to a Frobenius morphism $\mathbb{P}^{2} \rightarrow \mathbb{P}^{2}$. This completes the proof.

Remark. The image of each flex of $E$ under the Gauss map $\gamma: E \rightarrow E^{*} \subset \check{\mathbb{P}}^{2}$ is not a flex of $E^{*}$ if $j(E) \neq 0$.

\section{Proof of TheOREM 1}

Now we prove Theorem 1. Since the if part of the theorem is trivial, we give a proof of the only if part. We denote by $d_{i}$ the degree of $C_{i}$.

First we note that $d_{1}=d_{2}$. In fact, since Gauss maps $\gamma_{1}$ of $C_{1}$ and $\gamma_{2}$ of $C_{2}$ are purely inseparable by Kaji's theorem, we have that the geometric genus of $C_{i}$ coincides with that of $C_{i}^{*}$ for $i=1,2$. Hence we have $d_{1}=d_{2}$ because both plane curves are smooth and have the same genus.

Let $q_{i}$ be the (inseparable) degree of $\gamma_{i}$ for $i=1,2$. Since $C_{1}^{*}=C_{2}^{*}$ and $d_{1}=d_{2}$, we have $q_{1}=q_{2}$ by the Plücker formula. This means that the reflexivity of $C_{1}$ coincides with that of $C_{2}$. It is obvious that if both $C_{1}$ and $C_{2}$ are reflexive, then $C_{1}^{*} \neq C_{2}^{*}$. So we may assume that $C_{1}$ and $C_{2}$ are nonreflexive. 
Let us consider the correspondence

$$
G^{\prime}=\left\{(P, Q) \in C_{1} \times C_{2} \mid T_{P} C_{1}=T_{Q} C_{2}\right\}
$$

with reduced structure, where $T_{P} C$ means the embedded tangent line to a curve $C$ at a point $P \in C$. Obviously, the diagram

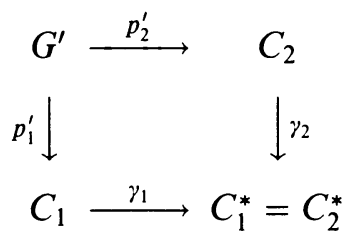

is commutative, where $p_{1}^{\prime}$ and $p_{2}^{\prime}$ are projections. Note that $p_{i}: G^{\prime} \rightarrow C_{i}$ $(i=1,2)$ are surjective by our assumption $C_{1}^{*}=C_{2}^{*}$ and generically one-toone by Kaji's theorem. Hence $\operatorname{dim} G^{\prime}=1$ and a one-dimensional component of $G^{\prime}$ is unique. We denote by $G$ the one-dimensional component of $G^{\prime}$ and by $p_{i}$ the restriction of $p_{i}^{\prime}$ to $G$.

We show that $p_{1}$ and $p_{2}$ are separable. Let us consider the commutative diagram of Zariski tangent spaces for a general point $(P, Q) \in G$ :

$$
\begin{array}{ccc} 
& & \mathscr{T}_{P} C_{1} \\
\text { 1st projection } & \nearrow & \uparrow_{d p_{1}} \\
\mathscr{T}_{(P, Q)} C_{1} \times C_{2}=\mathscr{T}_{P} C_{1} \times \mathscr{T}_{Q} C_{2} & \hookleftarrow & \mathscr{T}_{(P, Q)} G \\
\text { 2nd projection } & \searrow & \uparrow_{d p_{2}} \\
& & \mathscr{T}_{Q} C_{2}
\end{array}
$$

Therefore, $d p_{1} \neq 0$ or $d p_{2} \neq 0$. Hence, $p_{1}$ or $p_{2}$ is separable. Since $\gamma_{1} \circ p_{1}=$ $\gamma_{2} \circ p_{2}$ and $\gamma_{1}$ and $\gamma_{2}$ are purely inseparable of the same degree, both $p_{1}$ and $p_{2}$ are separable; therefore, both $p_{1}$ and $p_{2}$ are birational. Since $C_{1}$ and $C_{2}$ are smooth, $p_{1}$ and $p_{2}$ are isomorphisms. Let $\sigma=p_{2} \circ p_{1}^{-1}: C_{1} \stackrel{\sim}{\rightarrow} C_{2}$. We need the following

Lemma. There is an automorphism $\tilde{\sigma}$ of $\mathbb{P}^{2}$ such that the diagram

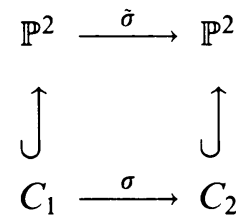

is commutative.

Proof. Since $C_{1}$ and $C_{2}$ are smooth plane curves, both curves are linearly normal, i.e., $H^{0}\left(\mathbb{P}^{2}, \mathscr{O}(1)\right) \rightarrow H^{0}\left(C_{i}, \mathscr{O}_{C_{i}}(1)\right)$ is an isomorphism. So it suffices to show that $\sigma^{*} \mathscr{O}_{C_{2}}(1) \cong \mathscr{O}_{C_{1}}(1)$. When $d \geq 4$, this is guaranteed by $[1$, p. 56 , Exercise 18] and when $d=2$, this is obvious.

Now we prove the claim when $d=3$ under our situation. Since $C_{1}$ and $C_{2}$ are nonreflexive smooth cubic, we have $p=2$. By Proposition 3, the map $C_{i} \stackrel{\gamma_{i}}{\rightarrow} C_{i}^{*} \stackrel{\gamma_{i}^{*}}{\rightarrow} C_{i}^{* *}$ sends the flexes of $C_{i}$ onto those of $C_{i}^{* *}$ for $i=1,2$. Since 
the diagram

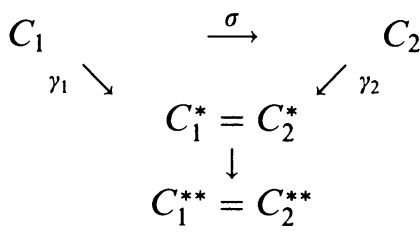

is commutative, $\sigma$ sends the flexes of $C_{1}$ onto those of $C_{2}$. Since $\mathscr{O}_{C_{i}}(1) \cong$ $\mathscr{O}_{C_{i}}(3 R)$ for each flex $R \in C_{i}$, we have $\sigma^{*} \mathscr{O}_{C_{2}}(1) \cong \mathscr{O}_{C_{1}}(1)$.

Let us return to the proof of Theorem 1. Let $P \in C_{1}$. Then $\tilde{\sigma}\left(T_{P} C_{1}\right)=$ $T_{\sigma(P)} C_{2}$. Since $T_{P} C_{1}=T_{\sigma(P)} C_{2}$ by our assumption, we have $\tilde{\sigma}\left(T_{P} C_{1}\right)=T_{P} C_{1}$. Consider the linear transformation of the dual plane $\check{\tilde{\sigma}}^{-1}: \check{\mathbb{P}}^{2} \rightarrow \check{\mathbb{P}}^{2}$. Then we have $\check{\tilde{\sigma}}^{-1}\left(\left(T_{P} C_{1}\right)^{*}\right)=\left(T_{P} C_{1}\right)^{*}$ for each $P \in C_{1}$. Hence we have that $\left\{\left(T_{P} C_{1}\right)^{*} \mid P \in C_{1}\right\}$ are collinear in $\check{\mathbb{P}}^{2}$ because $\check{\tilde{\sigma}}^{-1}$ is not the identity map. Therefore $C_{1}^{*}=C_{2}^{*}$ is a line. This means that $C_{1}$ and $C_{2}$ are conics in characteristic two with the same center by Lluis's theorem [9]. This completes the proof.

\section{SMooth PLANe CURVES With AN ASSIGNed Class}

The purpose of this section is to prove Theorem 2. Let $d^{*}$ be a given prime number and $C$ a smooth plane curve of degree $d$ with $\operatorname{deg} C^{*}=d^{*}$. By the Plücker formula, we have

$$
p^{e} d^{*}=d(d-1)
$$

where $p^{e}$ is the (inseparable) degree of the Gauss map of $C$.

First we consider the case $p \neq 2$. Since $d(d-1)$ is divisible by 2 , we have $d^{*}=2$ because $d^{*}$ is prime. On the other hand, since $p^{e} \leq d$, we have $2 d \geq d(d-1)$ by the Plücker formula. Hence we have $d=2$ or 3 . If $d=2$, then (1) occurs. If $d=3$, then $p^{e}=d=3$, but such a curve does not exist because there are no smooth curves with $p^{e}=d>2[4,(3.4)]$.

Next we consider the case $p=2$. If $e>1$, then $d=k \cdot 2^{e}+1$ by the generalization of Pardini's theorem [3, 5]. Hence we have

$$
d^{*}=k\left(k \cdot 2^{e}+1\right) \text {. }
$$

Since $d^{*}$ is prime, we have $k=1$ and $d^{*}=2^{e}+1$. In this case, (3) occurs by the latter part of the generalization of Pardini's theorem. If $e=1$, then we have $2 d^{*}=d(d-1)$. Since $d^{*}$ is prime, we have

$$
2=d-1 \text { and } d^{*}=d \text {, which is a prime number, }
$$

or

$$
2=d \text { and } d^{*}=d-1, \quad \text { which is a prime number. }
$$

In the former case, (2) occurs. The latter case does not occur because $d^{*}=1$. If $e=0$, then $d^{*}=d(d-1)$. Since $d^{*}$ is prime, we have $d=2$. So $C$ is a conic in characteristic two, but such a curve is nonreflexive, i.e., $e>0$. This is a contradiction.

\section{ACKNOWLEDGMENT}

The author would like to thank Akira Ohbuchi for helpful conversations, and to Steve Kleiman for pointing out an error in the references in the previous version. 


\section{REFERENCES}

1. E. Arbarello, M. Cornalba, P. A. Griffith, and J. Harris, Geometry of algebraic curves, Vol. I, Springer-Verlag, Berlin, Heidelberg, New York, and Tokyo, 1984.

2. M. Deuring, Invarianten und normalformen elliptischer funktionenkörper, Math. Z. 47 (1941), 47-56.

3. A. Hefez, Nonreflexive curves, Compositio Math. 69 (1989), 3-35.

4. M. Homma, Funny plane curves in characteristic $p>0$, Comm. Algebra 15 (1987), 14691501.

5. _ A souped-up version of Pardini's theorem and its application to funny curves, Compositio Math. 71 (1989), 295-302.

6. H. Kaji, On the Gauss maps of space curves in characteristic $p>0$, Compositio Math. 70 (1989), 177-197.

7. S. Kleiman, Tangency and duality, CMS Conf. Proc., vol. 6, Amer. Math. Soc., Providence, RI, 1986, pp. 163-226.

8. _ Multiple tangents of smooth plane curves (after Kaji), Contemp. Math., vol. 116, Amer. Math. Soc., Providence, RI, 1991, pp. 71-84.

9. E. Lluis, Variedades algebraicas con ciertas conditiones en sus tangentes, Bol. Soc. Mat. Mexicana (2) 7 (1962), 47-56.

10. A. Wallace, Tangency and duality over arbitrary fields, Proc. London Math. Soc. (3) 6 (1956), 321-342.

Department of Mathematics, Faculty of Education, Yamaguchi University, YAMAGUCHI 753, JAPAN

E-mail address: f00301@sinet.ad.jp

Current address: Department of Mathematics, Room 2-587, Massachusetts Institute of Technology, Cambridge, Massachusetts 02139

E-mail address: homma@math.mit.edu 